\title{
Absorption and Emission in the Non-Poisson Case: The Theoretical Challenge Posed by Renewal Aging
}

\author{
Gerardo Aquino, \\ Institute for Theoretical Physics, University of Amsterdam Valckenierstraat 65, \\ 1018 XE Amsterdam, The Netherlands
}

Luigi Palatella, Istituto dei Sistemi Complessi del CNR, Dipartimento di Fisica dell'Università di Roma "La Sapienza", P.le Aldo Moro 2, 00185 Roma, Italy

and Paolo Grigolini

Center for Nonlinear Science, University of North Texas, P.O. Box 311427, Denton, Texas 76203-1427

Istituto dei Processi Chimico Fisici del CNR Area della Ricerca di Pisa, Via G. Moruzzi 1,56124 Pisa, Italy

Dipartimento di Fisica dell'Università di Pisa and INFM, via Buonarroti 2, 56126 Pisa, Italy

Received on 6 January, 2005

\begin{abstract}
This short paper aims at clarifying the physical meaning of a previous pubblication [G. Aquino, P. Grigolini, L. Palatella, Phys. Rev. Lett. 93050601 (2004)], using later, although very recent, results. This has to do with the challenges posed to the Kubo-Anderson (KA) theory, and more in general to any form of Liouville-like approach, by the discovery of intermittent resonant fluorescence with a non-exponential distribution of waiting times. We show that to properly address the treatment of these problems the KA theory, valid in the case of aged systems, should be extended to aging systems, aging for a very extended time period or even forever, being a crucial consequence of non-Poisson statistics. This ambitious goal can be realized if we adopt the assumption that real wave-function collapses occur.
\end{abstract}

\section{Introduction}

In the last few years, as a consequence of an increasingly faster technological advance, it has become clear that the conditions of ordinary statistical mechanics assumed by the lineshape theory of Kubo and Anderson (KA) [1], are violated by some of the new materials. For instance, the experimental research work of Neuhauser et al. [2] has established that the fluorescence emission of single nanocrystals exhibits interesting intermittent behavior, namely, a sequence of "light on" and "light off" states, departing from Poisson statistics. In fact, the waiting time distribution in both states is non-exponential, and it shows a universal power law behavior [3]. In this paper, for simplicity, we assign to both states the same waiting time distribution

$$
\psi(t)=(\nu-1) \frac{T^{\nu-1}}{(t+T)^{\nu}},
$$

with $1<\nu<\infty$. The parameter $T>0$ is introduced for the purpose of making $\psi(t)$ finite at $t=0$ so as to ensure its normalization. We shall focus on the case when $\nu<3$. In accordance with Brokmann et al. [4], the experimental condition $\nu<2$ implies that the observed waiting time dis- tribution depends on the time at which observation begins. Let us assume that the probability of the first jump from the "on" ("off") to the "off" ("on") state is given by Eq. (1), if the observation begins at $t=0$. If the observation begins at a later time $t_{a}>0$, the distribution of the sojourn times, before the first jump, turns out to be different from Eq. (1): it is $t_{a}$ dependent and, for this reason, is denoted by $\psi_{t_{a}}(t)$. This notation reflects the fact that $\psi_{t_{a}}(t)$ is a property of the same kind as $\psi(t)$, obtained by setting the observation beginning at $t_{a}>0$, rather than at $t_{a}=0$. Consequently, $\psi(t)=\psi_{t_{a}=0}(t)$. Note that throughout the theoretical treatment of Section 5 we shall use the notation:

$$
f\left(t, t_{a}\right) \equiv \psi_{t_{a}}(t)
$$

According to renewal theory, in the non-Poisson case, $\psi_{t_{a}}(t)$ becomes slower and slower with increasing $t_{a}$ [4]. This is the property responsible for the breakdown of the ordinary KA theory: it is the aging effect on which we focus our attention in this paper. It is worth noticing that when $\nu>2$, this aging effect is still present, in a less dramatic form, given the fact that a stationary condition exists, even if the regression to it takes a virtually infinite time if $\nu<3$ [5]. 
As argued in Section 2, aging is a challenge to the theoretical treatment based on the ordinary KA approach. This is confirmed by the work of Ref. [6]. These authors showed how to derive the absorption lineshape in the case $\nu>2$, when the stationary condition applies, and evaluated the form that the spectrum would have, immediately after switching on the radiation field, when the non-stationary condition $\nu<2$ holds true. This means that, although remarkably interesting, this work left unsolved the problem of how to deal with aging dynamics. Here we illustrate a way to evaluate the time evolution of the absorption spectrum, so as to take into account the aging effects of Brokmann et al., with $\nu<2$, as well as those of Ref. [5], with $\nu>2$.

\section{Renewal aging}

The statistical analysis made by Brokmann et al [4] on the sequence of "light on" and "light off" signals gives clear indication of aging, thereby providing compelling evidence that blinking quantum dots are renewal processes. It is worth to stress the nature of aging with renewal processes [7]. An important point to discuss is the physical meaning of the brand new condition at $t=0$. The renewal theory was born for the practical purpose of solving probability problems connected with the failure and replacement of components, such as electric bulbs [7]. Let us imagine therefore a sequence $\left\{\tau_{i}\right\}$ obtained as follows. At time $t=0$ we switch on a brand new electric bulb. At time $t=\tau_{1}$ a failure occurs, and the electric bulb is immediately replaced by another one, totally identical, but brand new, lasting for a time $\tau_{2}$, and so on. To establish a connection with the physics of blinking quantum dots, we assume $\tau_{1}$ to denote the time duration of a "ligth on" state, $\tau_{2}$ to denote the time duration of a "light off" state, and so on. The prescription of alternating a "light off" to a "light on" state is correct, thanks to the assumption made in Section 1, about the statistical equivalence between "light on" and "light off" states. In conclusion, the second electric bulb, representing the "light off" state, fails at time $t=\tau_{1}+\tau_{2}$ and it is immediately replaced by a third one, representing the "light on" state. The third electric bulb fails at time $t=\tau_{1}+\tau_{2}+\tau_{3}$ and it is immediately replaced by a forth one, which represents the new "light off" state, and so on.

According to Cox [7], in addition to the waiting time distribution $\psi(t)$, which is here assumed to have the analytical form of Eq. (1), it is convenient to use also the survivor function $\Psi(t)$, defined by

$$
\Psi(t)=\int_{t}^{\infty} d t^{\prime} \psi\left(t^{\prime}\right)
$$

which, with $\psi(t)$ given by Eq. (1) gets the simple analytical expression

$$
\Psi(t)=\left[\frac{T}{(t+T)}\right]^{\nu-1} .
$$

Cox [7] introduces also the age-specific failure rate, $r(t)$, which is proven to be related to $\Psi(t)$ by

$$
r(t)=-\frac{1}{\Psi(t)} \frac{d \Psi(t)}{d t} .
$$

With the choice of Eq. (1) we get

$$
r(t)=\frac{r_{0}}{\left(1+r_{1} t\right)},
$$

where

$$
r_{0} \equiv \frac{(\nu-1)}{T}
$$

and

$$
r_{1} \equiv \frac{1}{T}
$$

We can alternativel say that the inverse power law index $\nu$ is determined by the ratio of the brand new material rate, $r_{0}$, to the dynamical rate, $r_{1}$, as follows

$$
\nu=1+\frac{r_{0}}{r_{1}} .
$$

These arguments shed light on the physical meaning of aging. The age-specific failure rate is time independent only in the Poisson case. In the case under discussion in this paper, in the time asymptotic limit the decay of the age-specific failure rate is proportional to $1 / t$. This means that there is no time scale, and that our choice of brand new initial condition, with all the electric bulbs at the beginning of their function, will not set any significant limitation to the generality of our conclusions. It is evident that even if we switch on the radiation field at a later time $t_{a}>0$, which is then assumed to be the time origin for the calculation of the absorption and emission spectrum, the time evolution of the corresponding spectra will not significantly depart from the results of this paper.

As we shall see hereby, and in Sections 4 and 5, we make the assumption that the laser excitation field is turned on at the same time when the Gibbs system is prepared in the brand new condition. In fact, to meet the ordinary requirement of referring the theoretical investigation to a Gibbs system, we create a virtually infinite number of sequences $\left\{\tau_{i}\right\}$, of the earlier described kind. This Gibbs system is monitored by means of the radiation field, with the condition that the radiation field is switched on at time $t=0$. This corresponds to the brand new condition, where all the "electric bulbs" are simultaneously switched on. The radiation field keeps monitoring the system dynamics from time $t=0$ on. For any system of the Gibbs ensemble, the radiation field acts at time $t>0$ upon an individual sequence, which is $t$ old. This is a challenging condition for a Liouville-like treatment, where the radiation field is expected to act on a density matrix, evolving from initial condition of a given age, rather than on a single trajectory whose age keeps changing with time.

Note that in the case where the sequence $\left\{\tau_{i}\right\}$ is used to mimic the fluorescence signal of a blinking quantum dot, we create a dichotomous fluctuation $\xi(t)$ with the convention that $\xi(t)=W$, when $t$ belongs to the laminar region of a "light on" signal, and $\xi(t)=-W$, when $t$ lies on a "light off" state. In this case, the correlation function of $\xi(t)$, denoted by $\Phi_{\xi}\left(t_{1}, t_{2}\right)$, is non-stationary [8]. In the case $\nu>2$, in spite of the property of Eq. (6), which suggests a 
perennial aging condition, at least ideally a stationary state can be defined, and with it a stationary correlation function $\Phi_{\xi}\left(t_{1}-t_{2}\right)$.

It is important to stress that a sequence with the analytical form of Eq. (1) can be created by using the modulation prescription proposed by Beck [9]. Note that this modulation prescription generates distributions of the form of Eq. (1), which is the same as that emerging from the non-extensive thermodynamics of Tsallis [10]. Thus, this modulation approach, under the name of superstatistics has been recently proposed as a way to account for the emergence in nature of any form of non-Poisson distribution as well as those of the Tsallis form $[11,12]$, which in turn was originally proposed to account for the increasing number of experimental observations

The authors of Ref.[13] have proved that in the case $\nu>2$, modulation (superstatistics) not only can yield for $\psi(t)$ the same analytical form as that of Eq. (1): In this case modulation generates for the stationary correlation function $\Phi_{\xi}(t)$ the same analytical form as that predicted by renewal theory. On the other hand, in this case the adoption of the ordinary Liouville-like procedure allows us to describe the time evolution of the electric dipole fluctuating back and forth from the the "light on" to the "light off" condition, using the density formalism [13]. Apparently this result leads to the conclusion that the methods used in the Poisson case [14] can be applied also to the non-Poisson condition. This is not quite correct. This extension is possible if the nonPoisson form emerges from modulation (superstatistics). It is not more legitimate if the non-Poisson properties under study are of genuinely renewal kind.

A transparent explanation of this important fact is given by the recent paper of Ref.[15]. The demonstration of Ref. [15] runs as follows. The construction of the Generalized Master Equation (GME) produced by dichotomous fluctuation is made possible by the factorization condition that, in the case with no bias, for the fourth-order correlation function reads:

$$
\left\langle\xi\left(t_{1}\right) \xi\left(t_{2}\right) \xi\left(t_{3}\right) \xi\left(t_{4}\right)\right\rangle=\left\langle\xi\left(t_{1}\right) \xi\left(t_{2}\right)\right\rangle\left\langle\xi\left(t_{3}\right) \xi\left(t_{4}\right)\right\rangle .
$$

The higher-order correlation functions are analogously defined [15]. The authors of Ref. [15] prove that in the renewal non-Poisson case, this factorization property is violated, thereby yielding the breakdown of the corresponding GME.

To a first sight, the definition of aging through the time dependent rate of Eq. (5) leads to the conclusion that any waiting time distribution departing from the exponential form yields aging. Actually, it is not so. Aging is a property of the distribution of first sojourn times. After the first exit, a rejuvenation effect occurs [16]. The dependence on time of the rate $r(t)$ is not, by itself, a compelling proof of aging. In fact, the non-Poisson form produced by modulation stems from the statistical averages over a distribution of Poisson rates, and the time dependence of $r(t)$ does not reflect the existence of a renewal aging. As a matter of fact, the waiting time distribution depends on the choice of observation time $t_{a}$. If $t_{a}>t=0, t=0$ being the time at which the brand new condition is realized, the corresponding waiting time distribution, $\psi_{t_{a}}(t)$, turns out to become slower and slower with increasing $t_{a}$. It has been recently proved [17] that in the case of infinitely slow modulation, the proper condition behind superstatistics, the function $\psi_{t_{a}}(t)$ is independent of $t_{a}$. In conclusion, superstatistics yields no aging, and can be ruled out as the correct perspective behind the physics of blinking quantum dots.

On the basis of the earlier remarks we conclude that the correct proposal for the emergence of a departure from the Poisson condition in the case of the blinking quantum dots must rest on renewal theory. An example of model fitting this important requirement is given by the work of Ref. [18]. This model, in turn, is formally equivalent to that proposed years ago by Bouchaud [19, 20], to explain the dynamics of glassy systems. These two models are significant examples of a wider category of renewal models. A very attractive model of this kind is the hierarchical trap model illustrated by Zaslavsky [21]. To explain in words this model, adopting an interpretation making it fit the physics of blinking quantum dots, we say that the electron makes a jump from a state where spontaneous emission of light is possible, to a state that can be thought of as the doorway state of a region where ligth emission is quenched. From this state the electron can jump either back or forward, the forward direction corresponding to a deeper and deeper embedding within the "ligth off" region. The forward and backward jumping rates become smaller and smaller with an increased embedding. The electron can come back to the doorway state and from there to the "ligth on" region. This generates a sequel of sojourn times in the "light off" state, with no correlation whatsoever among themselves. The process of slow modulation apparently fits the condition of no correlation among different sojourn times. Actually, in this case large portions of the sequence $\left\{\tau_{i}\right\}$ would correspond to drawing random numbers from the same Poisson distribution, thereby implying a subtle form of correlation.

In this paper we illustrate the procedure to follow when no direct recourse can be made to the density methods. This is a challenging request that we shall address in the next sections.

\section{The Kubo stochastic oscillator}

We use the following stochastic equation:

$$
\frac{d}{d t} \mu(t)=i\left(\omega_{0}+\xi(t)\right) \mu(t) .
$$

The quantity $\mu(t)$ is a complex number, corresponding to the operator $|e\rangle\langle g|$ of the more rigorous quantum mechanical treatment [22], $|e\rangle$ and $|g\rangle$ being the excited and the ground state, respectively, $\omega_{0}$ is the energy difference between the excited and the ground state, and $\xi(t)$ denotes the energy fluctuations caused by the cooperative environment of this system. The fluctuation $\xi(t)$ is derived from the sequences $\left\{\tau_{i}\right\}$ using the procedure described in Section 2. In the presence of the coherent excitation, Eq. (11) becomes

$$
\frac{d}{d t} \mu(t)=i\left(\omega_{0}+\xi(t)\right) \mu(t)+k \exp (i \omega t)
$$


where $\omega$ denotes the radiation field frequency. It is convenient to adopt the rotating-wave approximation. Let us express Eq. (12) by means of the transformation $\tilde{\mu}(t)=$ $\exp (i \omega t) \mu(t)$. After some algebra, we get a simple equation of motion for $\tilde{\mu}(t)$. For simplicity we denote $\tilde{\mu}(t)$ with the symbol $\mu(t)$ again, thereby making the resulting equation read:

$$
\frac{d}{d t} \mu(t)=i(\delta+\xi(t)) \mu(t)+k,
$$

where $\delta=\omega_{0}-\omega$. The reader can easily establish the connection between this picture and the stochastic Bloch equation of Ref. [22] by setting $\mu=v+i u$. Note that the three components of the Bloch vector in Ref. [22], $(u, v, w)$, are related to the rotating-wave representation of the density matrix $\rho, v$ and $u$ being the imaginary and the real part of $e^{-i \omega t} \rho_{g e}$, and $w$ being defined by $w \equiv\left(\rho_{e e}-\rho_{g g}\right) / 2$. Note that the equivalence with the picture of Ref. [22] is established by assuming the radiative lifetime of the excited state to be infinitely large and the Rabi frequency $\Omega \equiv k$ vanishingly small.

It is straightforward to integrate Eq. (13), thereby getting

$$
\mu(t)=k \int_{0}^{t} d t^{\prime} e^{i \int_{t^{\prime}}^{t} \xi\left(t^{\prime \prime}\right) d t^{\prime \prime}} e^{i \delta\left(t-t^{\prime}\right)},
$$

with the dipole $\mu=0$, when the exciting radiation is turned on. Now we have to address the intriguing issue of averaging Eq. (14) over a set of identical systems, in such a way as to take aging effects into account $[4,23,5]$. In fact the averaging process turns Eq. (14) into

$$
\langle\mu(t)\rangle=k \int_{0}^{t} d t^{\prime}\left\langle e^{i \int_{t^{\prime}}^{t} \xi\left(t^{\prime \prime}\right) d t^{\prime \prime}}\right\rangle_{t^{\prime}} e^{i \delta\left(t-t^{\prime}\right)},
$$

with the subscript $t^{\prime}$ denoting that the system, brand new at $t=0$, is $t^{\prime}$-old when we evaluate the corresponding characteristic function, thereby implying that the distribution of waiting times before the first jump, is not $\psi(t)$, and $f\left(t, t^{\prime}\right)$ has to be used instead.

\section{Numerical calculation}

Let us now move to the numerical evaluation of the average of Eq.(14). To this purpose we use the Gibbs ensemble described in Section 2. For clarity sake, let us see again the basic steps of this procedure. Following the distribution of Eq. (1), we run $N$ distinct sequences $\left\{\tau_{i}\right\}$, with $N \gg 1$. For any of these $N$ sequences the sojourn in one of the two states begins exactly a time $t=0$ and ends at time $t=\tau_{1}$. For $N / 2$ of these sequences we use the "light on" as initial condition, and for $N / 2$ the "light off" state. Let us consider the former type of trajectories for illustration purpose. The "light on" state begins at $t=0$ and ends at $t=\tau_{1}$, at which time the "light off" state begins, ending at time $t=\tau_{1}+\tau_{2}$, and so on. With this numerical method we produce a set of fluctuations $\xi(t)$. Then, we create a set of diffusion trajectories $\int_{t^{\prime}}^{t} \xi\left(t^{\prime \prime}\right) d t^{\prime \prime}$, and hence the set of exponentials $e^{i \int_{t^{\prime}}^{t} \xi\left(t^{\prime \prime}\right) d t^{\prime \prime}}$. Since all trajectories of this set are $t^{\prime}$-old, the resulting numerical average is automatically equivalent to evaluating $\left\langle e^{i \int_{t^{\prime}}^{t} \xi\left(t^{\prime \prime}\right) d t^{\prime \prime}}\right\rangle_{t^{\prime}}$ with the waiting time distribution $f\left(t, t^{\prime}\right)$. We point out that the number of photons emitted at time $t$ is determined by $N(t)=\left\langle\mu(t) \mu^{*}(t)\right\rangle$. It is straightforward to prove that the rate of photons emitted, namely, $R(t) \equiv d N / d t$, obeys the relation

$$
R(t)=2 k \operatorname{Re}\langle\mu(t)\rangle .
$$

Thus, we conclude that the real part of $\mu(t)$ can be used to denote either emission or absorption at time $t$. We note that the approximation ensuring the equivalence between our picture and Ref. [22], for large photon count, has also the effect of making the absorption identical to the emission spectrum.

Figure 1 illustrates the rate of absorption changing upon change of time, when $\nu=2.5$. We see that the spectrum changes from a Lorentzian shape centered at $\omega=\omega_{0}$ to a bimodal shape at later time, in accordance with earlier theoretical work [24, 25]. The authors of Ref. [24] studied the aged condition $f(t, \infty)$ with $2<\nu<3$, and found that the corresponding spectrum has two sharp peaks. Klafter and $\mathrm{Zu}-$ mofen proved that the same case with $f\left(t, t_{a}=0\right)=\psi(t)$ yields Lévy diffusion, thereby implying an exponential characteristic function, and consequently a Lorentzian spectrum. It is remarkable that our theoretical perspective establishes a connection between the prediction of Zumofen and Klafter, valid at short times, and that of Ref. [24], valid at large times. Remarkably, we evaluate numerically also the spectrum time evolution in the case $\nu<2$, where the process of aging keeps going on forever without ever reaching the stationary condition, which does not exist, in this case (see Fig. 2).

\section{Theoretical calculations}

Let us show now how to reproduce these numerical results with a proper theoretical treatment. Let us consider as an example a single trajectory starting a time $t^{\prime}$ with $\xi=+W$ and ending at time $t$ with the same positive value after $n$ switches of the variable $\xi$ between its two values $\pm W$. In this case the integrand of (14), has the following form:

$$
\exp \left[\alpha_{+}\left(t_{1}-t^{\prime}\right)+\alpha_{-}\left(t_{2}-t_{1}\right)+\ldots . .+\alpha_{+}\left(t-t_{n}\right)\right]
$$

where $\alpha_{ \pm}=i(\delta \pm W)$ and $n$ can only be even.

To determine the contribution to $\langle\mu(t)\rangle$ of Eq. (15) stemming from this kind of trajectories, we have to average the term (17) on the set of all possible sequences of the variable $\xi(t)$ running from $t^{\prime}$ to $t$ with $n$ switches, to sum over $n$ and then to carry out the integration on $t^{\prime}$.

To this purpose, as earlier stated, besides the probability density $\psi(\tau)$ of having an interval $\tau$ between two generic consecutive switching events, it is necessary to use $f\left(\tau, t^{\prime}\right)$, this being the conditional probability density that, fixed a time $t^{\prime}$, the first next switching event of the variable $\xi(t)$ occurs at time $t^{\prime}+\tau$. The result of this procedure turns the contribution to (15) into: 

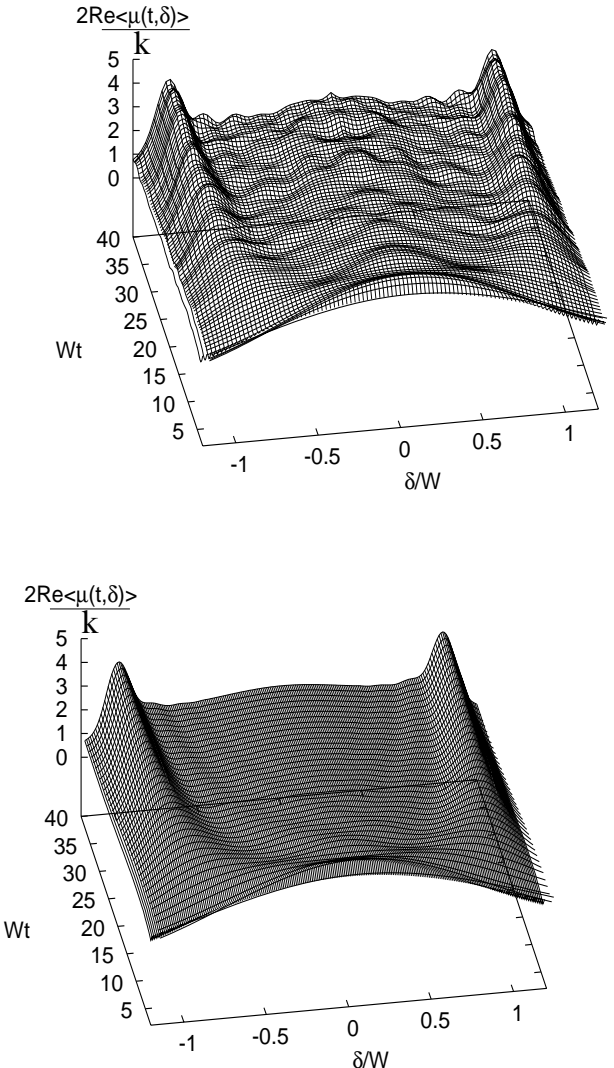

Figure 1. The numerical (top) versus the theoretical (bottom) absorption spectrum with $\nu=2.5, T \cdot W=0.4$. We assign to the fluctuation $\xi(t)$ the intensity $W=1$.

$$
\begin{aligned}
& \frac{k}{2} \int_{0}^{t} d t^{\prime}\left(F\left(t-t^{\prime}, t^{\prime}\right) e^{\alpha_{+}\left(t-t^{\prime}\right)}+\sum_{n=1}^{\infty} \int_{t^{\prime}}^{t} d t_{1} f\left(t_{1}-t^{\prime}, t^{\prime}\right)\right. \\
& \cdot e^{\alpha_{+}\left(t_{1}-t^{\prime}\right)} \int_{t_{1}}^{t} d t_{2} \psi\left(t_{2}-t_{1}\right) e^{\alpha_{-}\left(t_{2}-t_{1}\right)} \int_{t_{2}}^{t} d t_{3} \psi\left(t_{3}-t_{2}\right) \\
& \cdot e^{\alpha_{+}\left(t_{3}-t_{2}\right)} \ldots \int_{t_{2 n-1}}^{t} d t_{2 n} \psi\left(t_{2 n}-t_{2 n-1}\right) e^{\alpha_{-}\left(t_{2 n}-t_{2 n-1}\right)} \\
& \left.\cdot \Psi\left(t-t_{2 n}\right) e^{\alpha_{+}\left(t-t_{2 n}\right)}\right),
\end{aligned}
$$

where, in addition to the crucial probability density $f\left(\tau, t^{\prime}\right)$, we have used

$$
\Psi(\tau)=1-\int_{0}^{\tau} \psi\left(\tau^{\prime}\right) d \tau^{\prime}
$$

which is the conventional probability that no switch occurs for a generic interval of time $\tau$, and

$$
F\left(\tau, t^{\prime}\right)=1-\int_{0}^{\tau} f\left(\tau^{\prime}, t^{\prime}\right) d \tau^{\prime}
$$
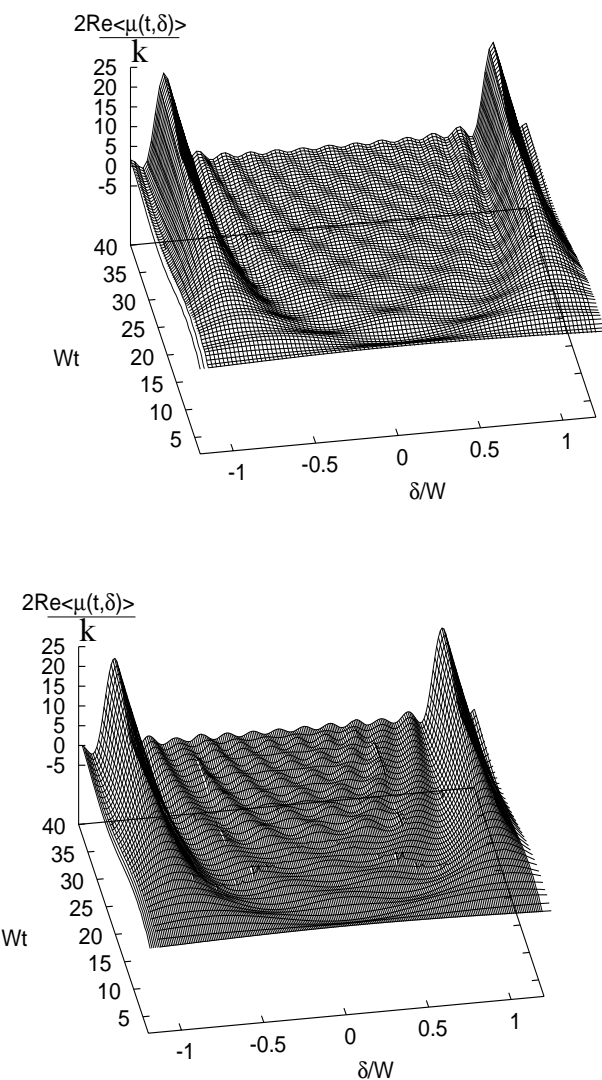

Figure 2. The numerical (top) versus the theoretical (bottom) absorption spectrum with $\nu=1.5, T \cdot W=0.4$. We assign to the fluctuation $\xi(t)$ the intensity $W=1$.

which is the corresponding aging property, depending on $f\left(\tau, t^{\prime}\right)$, and indicating therefore the conditional probability that, fixed $t^{\prime}$, no switch occurs between $t=t^{\prime}$ and $t=t^{\prime}+\tau$. The overall factor of $1 / 2$ of the contribution (18) is a consequence of the fact that at time $t^{\prime}$ the fluctuation $\xi(t)$, supposed to be positive, can get with the same probability the negative value. Let us address now the problem of finding an exact analytical expression for the crucial property $f\left(\tau, t^{\prime}\right)$. The exact expression for $f\left(\tau, t^{\prime}\right)$, is

$$
f\left(\tau, t^{\prime}\right)=\int_{0}^{t^{\prime}} d \tau^{\prime} G\left(t^{\prime}-\tau^{\prime}\right) \psi\left(\tau+\tau^{\prime}\right),
$$

where

$$
\begin{aligned}
& G(t) \equiv \delta(t)+\psi(t)+\sum_{n=2}^{\infty} \int_{0}^{t} d t_{1} \psi\left(t_{1}\right) \\
& \cdot \int_{t_{1}}^{t} d t_{2} \psi\left(t_{2}-t_{1}\right) \ldots \int_{t_{n-2}}^{t} d t_{n-1} \psi\left(t-t_{n-1}\right) .
\end{aligned}
$$

It is straightforward to find the Laplace transform of $G(t)$. This is given by

$$
\hat{G}(u)=\sum_{n=0}^{\infty} \hat{\psi}(u)^{n}=\frac{1}{1-\hat{\psi}(u)}
$$


where $\hat{\psi}(u)$ denotes the Laplace transform of $\psi(t)$. Thus, the Laplace transform of (21) with respect to $t^{\prime}$ reads:

$$
\hat{f}\left(\tau, u^{\prime}\right)=\frac{1}{1-\hat{\psi}\left(u^{\prime}\right)} e^{u^{\prime} \tau}\left[\hat{\psi}\left(u^{\prime}\right)-\int_{0}^{\tau} e^{-u^{\prime} y} \psi(y) d y\right],
$$

thereby yielding:

$$
f\left(\tau, t^{\prime}\right)=\mathcal{L}^{-1}\left[\hat{f}\left(\tau, u^{\prime}\right)\right] .
$$

where the double Laplace transform, after some algebra, reads

$$
\hat{f}\left(u, u^{\prime}\right)=\frac{\psi\left(u^{\prime}\right)-\psi(u)}{\left(u-u^{\prime}\right)\left[1-\psi\left(u^{\prime}\right)\right]} .
$$

With this expression the prescription necessary to evaluate the contribution to $\langle\mu(t)\rangle$ of Eq. (15) of the trajectories beginning in the "light on" state at $t^{\prime}$ and ending in the same state at $t$, is completed.

As a last step, we calculate the Laplace transform of (18), and of the equivalent expressions for all the other possible conditions of motion from $t^{\prime}$ to $t$, namely, with the noise $\xi(t)$ moving from $+W$ to $-W$, from $-W$ to $+W$ and, finally, from $-W$ to $-W$, as well. This procedure yields, as a final result, the Laplace transform of $R(t)$, denoted by $\hat{R}(u)$, which is proved to have the following analytical expression:

$$
\hat{R}(u)=k \mathcal{L}\left[\left\langle\mu(t)+\mu^{*}(t)\right\rangle\right]=\frac{k^{2}}{2}\left(A_{+}(u)+A_{-}(u)+C(u)\right) .
$$

The explicit expression for $A_{+}(u)$, which is calculated taking into account the contribution to $\langle\mu(t)\rangle$ of those trajectories ending at time $t$ with a positive value $\xi(t)=+W$ for the flucuating variable, is

$$
A_{+}(u) \equiv \frac{\hat{\Psi}\left(u-\alpha_{+}\right)\left[\hat{f}_{+}(u) \hat{\psi}\left(u-\alpha_{-}\right)+\hat{f}_{-}(u)\right]}{1-\hat{\psi}\left(u-\alpha_{+}\right) \hat{\psi}\left(u-\alpha_{-}\right)}+\hat{F}_{+}(u),
$$

with:

$$
\begin{aligned}
\hat{\Psi}\left(u-\alpha_{ \pm}\right) & =\mathcal{L}\left[\Psi(t) e^{\alpha_{ \pm} t}\right]=\frac{1-\hat{\psi}\left(u-\alpha_{ \pm}\right)}{u-\alpha_{ \pm}} \\
\hat{f}_{ \pm}(u) & \equiv \mathcal{L}\left[\int_{0}^{t} d t^{\prime} f\left(t-t^{\prime}, t^{\prime}\right) e^{\alpha_{ \pm}\left(t-t^{\prime}\right)}\right] \\
& =\frac{\hat{\psi}(u)-\hat{\psi}\left(u-\alpha_{ \pm}\right)}{-\alpha_{ \pm}(1-\hat{\psi}(u))}
\end{aligned}
$$

and

$\hat{F}_{ \pm}(u) \equiv \mathcal{L}\left[\int_{0}^{t} d t^{\prime} F\left(t-t^{\prime}, t^{\prime}\right) e^{\alpha_{ \pm}\left(t-t^{\prime}\right)}\right]=\frac{1 / u-\hat{f}_{ \pm}(u)}{\left(u-\alpha_{ \pm}\right)}$

$A_{-}(u)$ takes into account the contribution of all trajectories ending at time $t$ with the negative value $\xi(t)=-W$, for the fluctuating variable and is derived from the expression for $A_{+}(u)$ by replacing everywhere $\alpha_{ \pm}$with $\alpha_{\mp}$ (sending $\left.\hat{f_{ \pm}}, \hat{F_{ \pm}} \rightarrow \hat{f_{\mp}}, \hat{F_{\mp}}\right)$. Thus, $k\left(A_{+}(u)+A_{-}(u)\right) / 2$ represents the laplace transform of $\langle\mu(t)\rangle$. Finally $k C(u) / 2$ is the Laplace transform of $\left\langle\mu^{*}(t)\right\rangle$, and it is derived from the earlier expression for $k\left(A_{+}(u)+A_{-}(u)\right) / 2$ by replacing everywhere $\alpha_{ \pm}$with $-\alpha_{ \pm}$. To establish a comparison with the result of the numerical experiment we have anti-Laplace transformed the analytical expression of Eq. (27) using a Talbot algorithm implemented on Mathematica 5.0. The result of this procedure is illustrated by both Fig. 1 and Fig. 2, where the analytical predictions are compared to the corresponding numerical experiments. The overall qualitative agreement is remarkably good. Apparently, in Fig. 1 the departure of the theoretical calculation from the fine structure of the numerical result is larger than in Fig. 2. Actually, the numerical algorithm produces fluctuations of the same intensity in both cases, although the more detailed scale of Fig. 1 makes them appear larger. To establish beyond any doubt also the quantitative agreement, in Fig. 3 we compare the two predictions for specific values of the de-tuning parameter $\delta$. This shows that also the quantitative agreement is very satisfactory. Furthermore, when a stationary condition exists, it is straightforward to prove that Eq. (27) recovers, as asymptotic-time limit, the same result as that of [6].

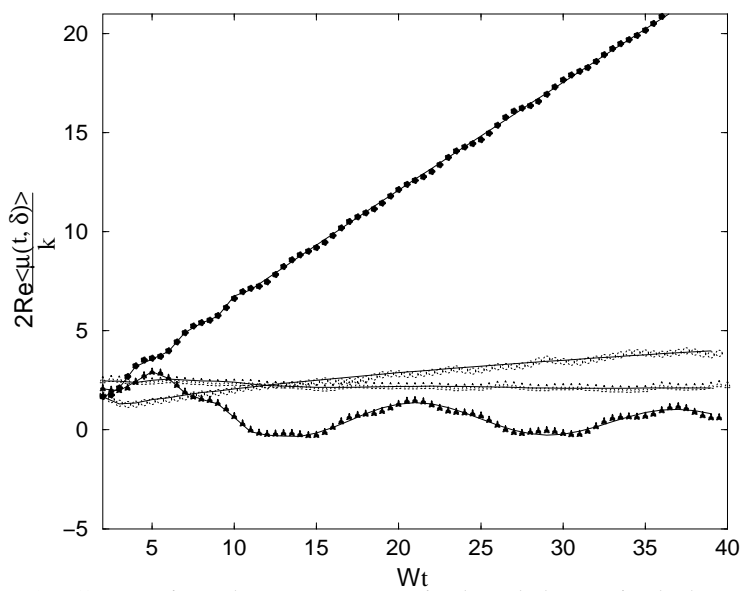

Figure 3. Comparison between numerical and theoretical absorption spectra for different values of the de-tuning parameter $\delta$ and of the power index $\nu$. Moving from the top to the bottom in the right hand portion of the figure, $\delta=1, \nu=1.5, \delta=1, \nu=2.5$, $\delta=0.6, \nu=2.5, \delta=0.6, \nu=1.5$. The numerical curves are denoted by black circles, open circles, open triangles and black triangles, respectively, and in this scale are almost indistinguishable from the full lines denoting the corresponding theoretical results.

\section{Conclusions}

We have adopted a procedure based on the observation of single trajectories, along the lines of the pioneering work of Montroll and Weiss on the Continuous Time Random Walk (CTRW) [26]. Rather than building up a master equation, we have studied the action of the radiation field on the single diffusional trajectory. Note that the field acting at time $t^{\prime}$ makes the photon emission depend on the state of a $t^{\prime}$-old trajectory. This essential single-system property is difficult, if not impossible, to faithfully reproduce with the density formalism. It is remarkable that this beneficial property of 
the procedure here adopted, yields Eq. (15), with the subscript $t^{\prime}$ working as an age indicator. This formula intertwines the system to the radiation field, thereby violating the ordinary linear response prescription, which is recovered in fact in the Poisson case. The breakdown of the linear response theory is a manifestation of the failure of the density method. A GME, of whatsoever origin, including that generated by the condition of being totally equivalent to the CTRW [27], always incorrectly responds to an external perturbation, as a consequence of missing, by its very nature, the entanglement with the external perturbation. It is important to notice that the breakdown of the density approach method has been independently observed by Sokolov, Blumen and Klafter [28]. These authors have built up a FokkerPlanck (FP)-like equation that should be equivalent to the CTRW of a given age (the brand new age, in their case). The action at later times of an external perturbation on this FP equation yields result that are distinctly different from those obtained by adopting the single trajectory picture with the external trajectory acting on the single unperturbed trajectory.

It is important to stress that we have adopted a vision corresponding to assuming the wave-function collapses to really occur. It is well known, in fact, that according to decoherence theory [29] the wave-function collapse is mimicked by a density matrix becoming diagonal in the basis set of the eigenstates of the variable that is measured [30]. This approach implies the adoption of a reduced density matrix, and consequently of the GME that one can derive from a Liouville equation, via the adoption of a convenient projection method (see, for instance, Ref. [31]). We have adopted, on the contrary, a picture based on the assumption of the existence of real trajectories. It is of fundamental importance at this stage to assess whether an equivalent Liouville or Liouville-like approach can be used, to yield the same result. The arguments of Section 2 seem to rule out this possibility.

GA and PG thankfully acnowledge ARO and Welch Foundation for financial support through Grant DAAD1902-1-0037 and 70525, respectively .

\section{References}

[1] R. Kubo, J. Phys. Soc. Japan 9, 935 (1954); Adv. Chem. Phys. 15, 101 (1969); P.W. Anderson, J. Phys. Soc. Jpn. 9, 316 (1954).

[2] R. G. Neuhauser, K. T. Shimizu, W. K. Woo, S. A. Empedocles, and M. G. Bawendi, Phys. Rev. Lett. 85, 3301 (2000).

[3] M. Kuno, D.P. Fromm, H. F. Hamann, A. Gallagher, and D.J. Nesbitt, J. Chem. Phys. 112. 3117 (2000).
[4] X. Brokmann et al, Phys. Rev. Lett. 90, 120601 (2003).

[5] P. Allegrini, G. Aquino, P. Grigolini, L. Palatella, and A. Rosa, Phys. Rev. 68, 056123 (2003).

[6] Y.-J. Jung, E. Barkai, R. J. Silbey, Chem. Phys. 284, 181 (2002).

[7] . D.R. Cox, Renewal Theory, Chapman and Hall, London (1962).

[8] P. Allegrini, G. Aquino, P. Grigolini, L. Palatella, A. Rosa, and B. J. West, arXiv:cond-mat/0409600.

[9] C. Beck, Phys. Rev. Lett. 87, 180601 (1-4) (2002).

[10] C. Tsallis, J. Stat. Phys. 52, 479 (1988).

[11] C. Beck, E.G.D. Cohen, Physica A 322, 267 (2003).

[12] C. Beck, Physica A 342, 139 (2004).

[13] M. Bologna, P. Grigolini, M. G. Pala, L. Palatella, Chaos, Solitons \& Fractals, 17, 601 (2003).

[14] P. Grigolini, Chem. Phys. 38, 389 (1979).

[15] P. Allegrini, P. Grigolini, L. Palatella, and B. J. West, Phys. Rev. E 70, 046118 (2004).

[16] G. Aquino, M. Bologna, P. Grigolini, and B. J. West, Phys. Rev. E 70, 036105 (2004).

[17] P. Grigolini, submittted to Physica A.

[18] R. Veberk, A. M. van Ojien, and M. Orrit, Phys. Rev. E B 66, 233202 (2002).

[19] J.P. Bouchaud, J. Phys. I (France) 2, 1705 (1992).

[20] J. P. Bouchaud and D.S. Dean, Aging on Parisi's Tree, J. Phys. I France 5, 265 (1995).

[21] G.M. Zaslavsky, Phys. Rep. 371, 461 (2002)

[22] Y.-J. Jung, E. Barkai, and R. Silbey, Adv. Chem. Phys. 123, 199 (2002) (see also: arXiv::cond-mat/0311428).

[23] E. Barkai, Phys. Rev. Lett. 90, 104101 (2003).

[24] P. Allegrini, P. Grigolini, B.J. West, Phys. Rev. E 54, 4760 (1996).

[25] J. Klafter, G. Zumofen, Physica A, 196, 102 (1993).

[26] E.W. Montroll and G.H. Weiss, J. Math. Phys. 6, 167 (1965).

[27] V. M. Kenkre, E.W. Montroll, and M.F. Shlesinger, J. Stat. Phys. 9, 45 (1973).

[28] I.M. Sokolov, A. Blumen and J. Klafter, Europhys. Lett. 56, 175 (2001).

[29] H.D. Zeh, Found. Phys. 1, 69 (1970).

[30] Z.H. Zurek, Phil. Trans. Roy. Soc. Lond. A 356, 1793 (1998).

[31] P. Grigolini, Adv. Chem. Phys. 62, 1 (1985). 